\title{
Rapamycin provides anti-epileptogenic effect in a rat model of post-traumatic epilepsy via deactivation of mTOR signaling pathway
}

\author{
FENG WANG* ${ }^{*}$ FUXIANG CHEN* ${ }^{*}$, GENBO WANG, SHUSHAN WEI, \\ FU FANG, DEZHI KANG and YUANXIANG LIN
}

Department of Neurosurgery, The First Affiliated Hospital of Fujian Medical University, Fuzhou, Fujian 350005, P.R. China

Received June 17, 2017; Accepted March 5, 2018

DOI: $10.3892 /$ etm.2018.6004

\begin{abstract}
The mammalian target of rapamycin (mTOR) signaling pathway has attracted much attention in recent years. However, the contribution of mTOR activation to the development of post-traumatic epilepsy (PTE) remains largely unknown. The purpose of the present study was to investigate the activation of mTOR signaling in a rat model of $\mathrm{FeCl}_{2}$-induced PTE, and to explore the potential effect of its specific inhibitor rapamycin. The results indicated that the expression levels of p-mTOR and p-P70S6K, the overactivation biomarkers of mTOR signaling, increased significantly in hippocampal and perilesional cortex following PTE induction. Notably, they were significantly decreased in the aformementioned brain regions following rapamycin treatment. Furthermore, the frequency and number of behavioral seizures and epileptic brain injury were also greatly reduced. These results suggest that hyperactivation of the mTOR signaling pathway is a crucial mechanism of PTE development, and it may be considered a novel therapeutic target for PTE treatment.
\end{abstract}

Correspondence to: Dr Yuanxiang Lin or Dr Feng Wang, Department of Neurosurgery, The First Affiliated Hospital of Fujian Medical University, 20 Chazhong Road, Fuzhou, Fujian 350005, P.R. China

E-mail: linyuanxiang1234@163.com

E-mail: 15005018772@163.com

"Contributed equally

Abbreviations: mTOR, mammalian target of rapamycin; PTE, post-traumatic epilepsy; TBI, traumatic brain injury; AEDs, anti-epileptic drugs; NS, normal saline; HRP, horseradish peroxidase; PBS, phosphate buffer

Key words: post-traumatic epilepsy, mammalian target of rapamycin, rapamycin, traumatic brain injury, seizures

\section{Introduction}

Post-traumatic epilepsy (PTE) is a common type of acquired epilepsies secondary to traumatic brain injury (TBI), accounting for approximately $10-25 \%$ of patients with moderate to severe injuries (1). After TBI, the brain carries out a series of pathological processes, including neuronal loss, gliosis, axonal sprouting, neurogenesis, inflammatory response, neurotransmitter release, and mitochondrial dysfunction, all of which have been connected to the epileptogenesis (2,3). Evidence indicate that the risk of PTE increases obviously 10 years after TBI and it usually develops refractory to medical management (4). At present, there are still many difficulties in PTE treatment partially because of the pathological mechanism has not been fully illustrated.

The mammalian target of rapamycin (mTOR) signaling pathway has been investigated extensively in recent decades. Experimental data suggest that mTOR pathway is involved in a number of physiological processes including cell proliferation, protein synthesis and cortical development (5-8). However, in recent years, it has also been reported to implicate in several neurological diseases, such as epilepsy (9). Evidences indicate that the mTOR pathway is activated in multiple type of epilepsies. For instance, epileptogenesis of cortical dysplasia, a common type of pediatric epilepsies, has been attributed to the over activation of mTOR signaling (9), which is also proven to involve in the development of temporal lobe epilepsy, the most common epilepsy in adults (10). The activation of mTOR protein kinase is regulated by multiple signaling pathways including PI3K/AKT, Ras/MAPK and AMPK. Phosphorylation level of downstream protein, such as p70 ribosomal S6 kinase (P70S6K), increased notably when the mTOR signaling is triggered (11). Up to now, only few research concentrates on the relationship between the development of PTE and the activation of mTOR. Although previous study demonstrated that mTOR-mediated epileptogenesis was activated via PI3K-Akt signaling in vitro model of PTE (12). However, they did not further explore the activation status of mTOR in animal model of PTE, nor did others.

Anti-epileptic drugs (AEDs) is the predominant therapy for epilepsy patients, however, about $30 \%$ of them are not 
sensitive to the treatment. Similarly, therapeutic effects of AEDs on patients with PTE is close to 35\% (13). Additionally, some patients even suffer from side effects caused by AEDs such as nausea, dizziness, lethargy and weight gain. Therefore, it is essential to find a novel therapeutic target for epilepsy patients. Fortunately, rapamycin, a macrolide antibiotic drug, has been demonstrated effectively via deactivation of mTOR signaling and then provides neuroprotective effect against epileptic injury in previous studies $(9,14,15)$. Furthermore, a published study also confirmed that administration of rapamycin also reduced epileptic activity, cell death and axon sprouting in organotypic hippocampal culture model of PTE (12).

Therefore, we performed this study to explore whether there is a abnormal activation of mTOR signaling in a rat model of PTE, and to determine the potential effect of rapamycin on mTOR-mediated epilepsic injury, aiming to find a novel therapeutic target for PTE management.

\section{Materials and methods}

Animals. Male Sprague-Dawley rats weighing between 180-220 $\mathrm{g}$ were purchased from the SLAC Laboratory Animal Co., Ltd. (Shanghai, China). The rats were housed in a $12 \mathrm{~h}$ of light and dark cycle, and were allowed free access to water and food. All the procedures performed were approved by the Animal Care and Use Committee of Fujian Medical University and in accordance with the guidelines of National Institutes of Health.

Establishment and assessment of PTE model. Procedures to induce the rat model of PTE has been described detailedly in previous studies $(16,17)$. Briefly, rats were anaesthetized with an intraperitoneal injection of chloral hydrate $(350 \mathrm{mg} / \mathrm{kg})$ and placed in a stereotaxic frame. Animals were injected with a $10 \mu 1$ of $100 \mathrm{mM} \mathrm{FeCl}_{2}$ or an equal volume of normal saline (NS) at a rate of $1 \mu 1$ per minute through a small burr hole with the following coordinates: $2.0 \mathrm{~mm}$ anterior, $3 \mathrm{~mm}$ lateral to bregma, $2 \mathrm{~mm}$ depth. The sham-operated group was inserted a neddle without receiving any solution. After the operation, the rats were kept in single cage. An experienced observer who was blinded to the experimental protocols graded the scale of seizures using modified Racine criteria, in which seizure stages were identified technically according to animal behaviors (18). Grade evaluation began at 1-2 $\mathrm{h}$ after the surgery.

Experimental design and pharmacological administration. In experiment 1, 129 rats were divided into three groups: Sham group $(\mathrm{n}=10)$, NS group $(\mathrm{n}=52)$, and PTE group $(\mathrm{n}=67)$. Brain tissues were collected at baseline, 1, 24 h, 1, 2 and 4 weeks, respectively. Each time point contained 10 rats. The expression levels of p-mTOR and p-P70S6K were determined using western blotting and immunohistochemistry. In experiment 2, 127 rats were randomly assigned to two groups: the $\mathrm{PTE}+$ vehicle group $(\mathrm{n}=62)$ and the PTE + rapamycin group $(\mathrm{n}=65)$. For drug administration, rapamycin was dissolved in solution containing 5\% Tween 80, 5\% PEG 400 and $4 \%$ ethanol, and then administered intraperitoneally at a dose of $6 \mathrm{mg} / \mathrm{kg}$ subsequently after induction of PTE. The time and

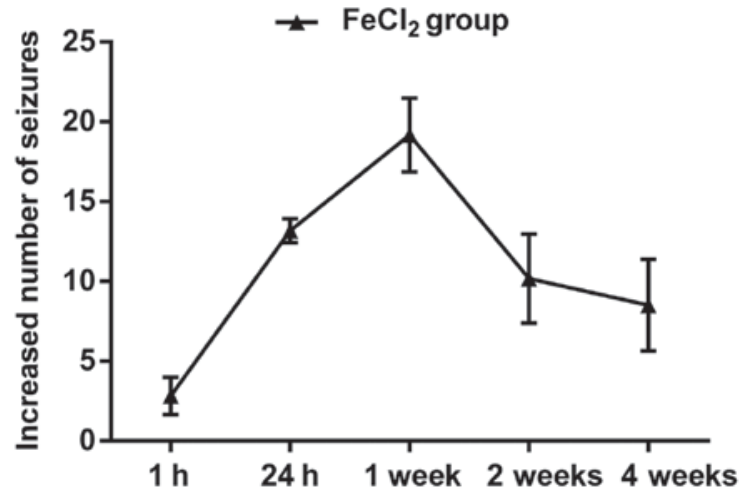

Figure 1. The number of behavioral seizures in rats subjected to $\mathrm{FeCl}_{2}$ injection. The frequency and number of behavioral seizures increased beginning at $1 \mathrm{~h}$ and sustained at least four weeks after the development of post-traumatic epilepsy.

dose of rapamycin injection was based on previous studis with slight modifications (19). The vehicle group received an equal volume of solution. The potential role of rapamycin was estimated also by western blotting and immunohistochemistry. In addition, the frequency and number of behavioral seizures were also evaluated.

Western blot analysis. Rats were decapitated separately at each time point after operation. Perilesional cortex and ipsilateral hippocampus were collected and processed for western blot assays as previously described (20). Protein concentrations were measured with a BCA kit (Beyotime, China). Equal amounts of protein were run on $10 \%$ SDS-PAGE and then transferred to polyvinylidene fluoride membranes at $100 \mathrm{~V}$ for $70 \mathrm{~min}$. After blocking with 5\% nonfat dry milk in PBS for $2 \mathrm{~h}$ at room temperature, the membranes were incubated overnight at $4^{\circ} \mathrm{C}$ with primary antibodies for rabbit anti-p-mTOR (1:200; Wuhan Boster Biological Technology, Ltd., Wuhan, China) and mouse anti-p-P70S6K (1:100; Wuhan Boster Biological Technology, Ltd.). The membranes were washed with PBST and further incubated with horseradish peroxidase (HRP) conjugated secondary antibodies at room temperature for $1 \mathrm{~h}$. Enhanced chemiluminescence reagent kit was used to detected the band' reactivitiy according to the manufacturer' protocol (Beyotime Institute of Biotechnology, Haimen, China). Band densities were quantified with ImageJ software (http://rsb.info.nih.gov/ij/). The results are presented as the relative densities to $\beta$-actin.

Immunohistochemistry. Immunohistochemistry was carried out as previously described (21). Briefly, rats were anaesthetized with chloral hydrate and perfused transcardially with saline followed by $4 \%$ paraformaldehyde in phosphate-buffered saline (PBS; 0.1 M, pH 7.4). After postfixation and dehydration, the brains were embedded in paraffin and cut into $4 \mu \mathrm{m}$ slices. The sections were deparaffinized and rehydrated in graded concentrations of ethanol to distilled water. and were further received antigen retrieval. Sections were incubated with $3 \% \mathrm{H}_{2} \mathrm{O}_{2}$ in PBS for 10 min to block the endogenous peroxidase activity. After blocking with 5\% fetal bovine serum in PBS, the sections were incubated with primary antibodies overnight at $4^{\circ} \mathrm{C}$. Next day, the sections were incubated with HRP-conjugated second antibodies at room temperature 

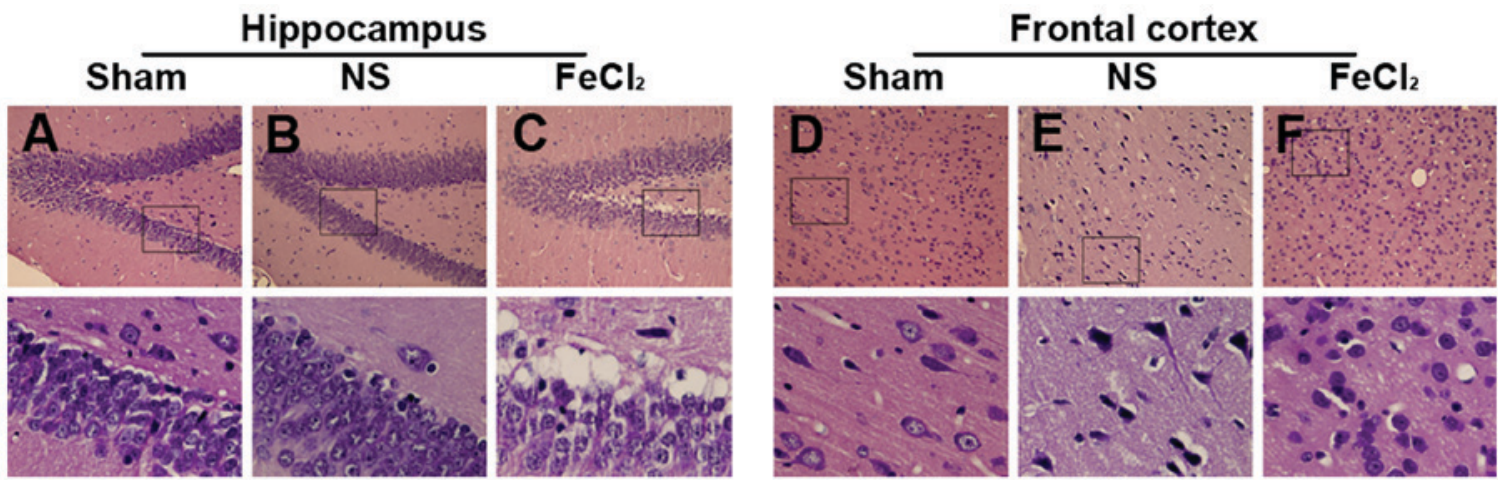

Figure 2. Hematoxylin and eosin of hippocampus and frontal cortex. (A) Cells in dentate gyrus show normal arrangements and distributions in the sham group. (B) Slight hippocampal changes in the NS-injected rats including neuronal degeneration, nuclear pyknosis and granule cells proliferation. (C) Hippocampal changes in the $\mathrm{FeCl}_{2}$-injected rats including plenty of neuronal loss, swelling, degeneration and death, as well as inflammatory cell infiltration and glial cell proliferation. (D) Cells in frontal lobe show normal arrangements and distributions in the sham group. (E) Slight cellular changes in frontal lobe of the NS-injected rats including neuronal degeneration, nuclear pyknosis and granule cells proliferation. ( $\mathrm{F}$ ) $\mathrm{Cellular}$ changes in frontal lobe of the FeCl${ }_{2}$-injected rats including plenty of neuronal loss, swelling, degeneration and death, as well as inflammatory cell infiltration and glial cell proliferation. Bottom graphs are enlarged view of the boxs above (magnification, x400), respectively. NS, normal saline.

for $1 \mathrm{~h}$. After that, diaminobenzidine and hematoxylin were used to stain successively. Sections were observed under an Olympus microscope and five non-overlapping fields (magnification, x400) per section were selected. Three sections per rat were used for quantification. Immunoreactivity densities were analyzed with ImageJ software by an investigator blinded to the treatment groups.

Statistical analysis. Data are presented as means \pm SD. The statistical analyses were performed using SPSS 13.0 (SPSS, Inc., Chicago, IL, USA). Student's t-test was used for comparison between groups. For behavioral data, Kruskal-Wallis test was applied. $\mathrm{P}<0.05$ was considered to indicate a statistically significant difference.

\section{Results}

General condition and epilepsy evaluation. A total of 194 rats received intracortical injection of $\mathrm{FeCl}_{2}$, and 150 of 176 (85.2\%) developed spontaneous behavioral seizures, including mouth and facial movement, head nodding, stiff tail, forelimb contraction and full motor seizures. In general, the frequency and number of behavioral seizures increased beginning at $1 \mathrm{~h}$ and peaked at 3-4 h. About $24 \mathrm{~h}$ later, generalized seizures almost dispeared, however, focal seizures could still be seen in most of the $\mathrm{FeCl}_{2}$-injected rats. Over the next four weeks, epileptic duration and frequency decreased continuously, in which rats primarily performed shivering, head nodding, facial movements and few limb clonus (Fig. 1). Additionally, 18 rats subjected to $\mathrm{FeCl}_{2}$ injection died during the observation, of which 10 rats died for status epilepticus, 8 rats for high seizures frequency. The remaining 26 rats failed to develop behavioral seizures and were excluded from final analyses. For animals injected with NS, only three rats developed focal seizures including head nodding and facial movements, but none of these rats died (data not shown). As expected, none of rats died or showed abnormal performance in the sham group.

Cortical and hippocampal injury in rats subjected to $\mathrm{FeCl}_{2}$ injection. As shown in Fig. 2A, granule cells, molecular and polymorphic layer of the dentate gyrus in the sham group showed normal arrangements and distributions. Moreover, there was also no neuronal degeneration or cell loss in frontal cortex (Fig. 2D). Slight cellular changes in frontal lobe and hippocampus were found in the NS-injected rats including neuronal degeneration, nuclear pyknosis and granule cells proliferation (Fig. 2B and E). However, more obvious cellular changes in perilesional cortex and hippocampus were confirmed in the $\mathrm{FeCl}_{2}$-injected rats than that in the NS-injected rats, such as plenty of neuronal loss, swelling, degeneration and death, as well as inflammatory cell infiltration and glial cell proliferation (Fig. 2C and F).

Increased expression of $p-m T O R$ and $p$-P70S6K in rats after intracortical injection of $\mathrm{FeCl}_{2}$. To examine whether mTOR signaling was upregulated in rats after PTE induction, we first carried out a temporal course of experiment with western blotting. As visible in Fig. 3A and B, at baseline, there was a small amount of p-mTOR and p-P70S6K expression in frontal lobe and hippocampus. Of note, both proteins increased significantly at $1 \mathrm{~h}$ after the surgery ( $\mathrm{FeCl}_{2}$ or NS injection) and peaked at 1 week in the abovementioned regions. Then protein levels of p-mTOR and p-P70S6K decreased to the bottom at 2 weeks (except p-mTOR expression in frontal cortex) while elevated again at 4 weeks (Fig. 3C-F). Furthermore, compared with rats injected with NS, perilesional and hippocampal p-mTOR levels in rats after PTE induction were significantly increased at all time points. Similarly, in addition to p-P70S6K expression in hippocampus at 2 week after surgery, p-P70S6K levels at each time point were higher than those in the NS-injected rats. Consistence with the results of western blotting, immunohistochemistry further demonstrated the higher increased levels of p-mTOR and p-P70S6K in frontal lobe and hippocampus following PTE (Fig. 4). Moreover, both proteins primarily expressed in cytoplasm.

Rapamycin reduced behavioral seizures and attenuated epileptic injury. Similar pattern of seizures was found in the vehicle group as compared with the PTE + rapamycin group, but actually, the PTE rats exerted significant decline of behavioral seizures ranging from $24 \mathrm{~h}$ to 4 weeks after treatment 
A
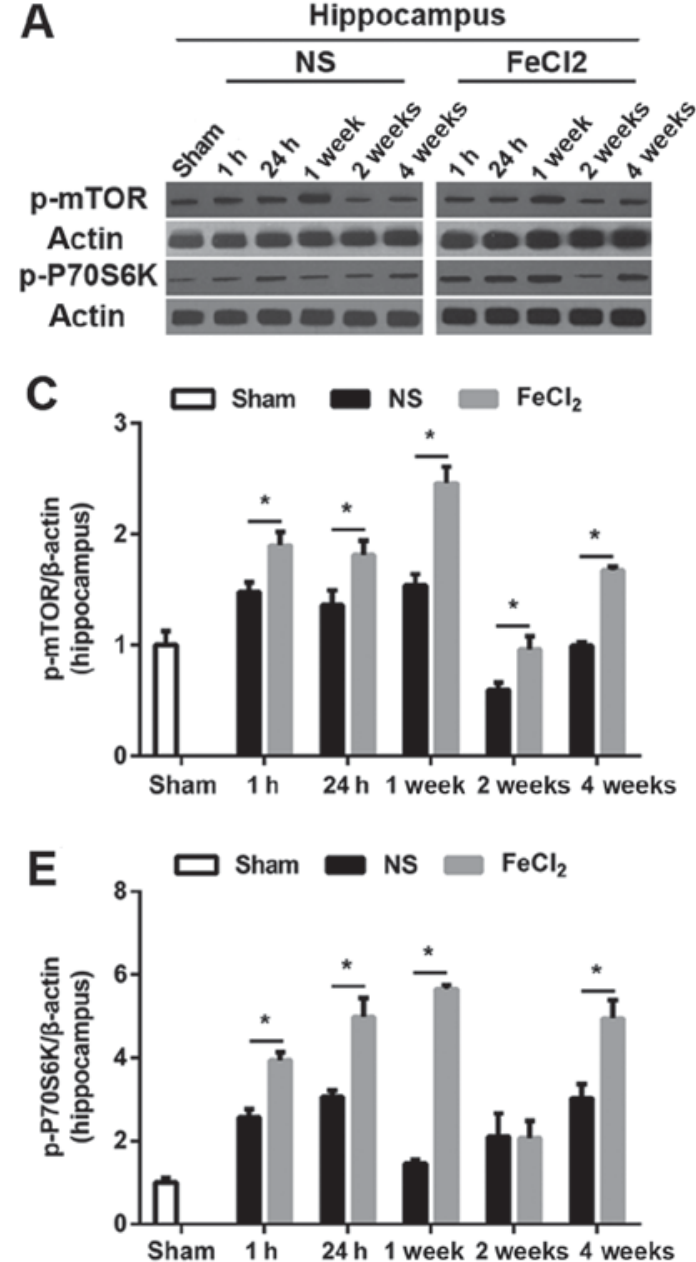

B
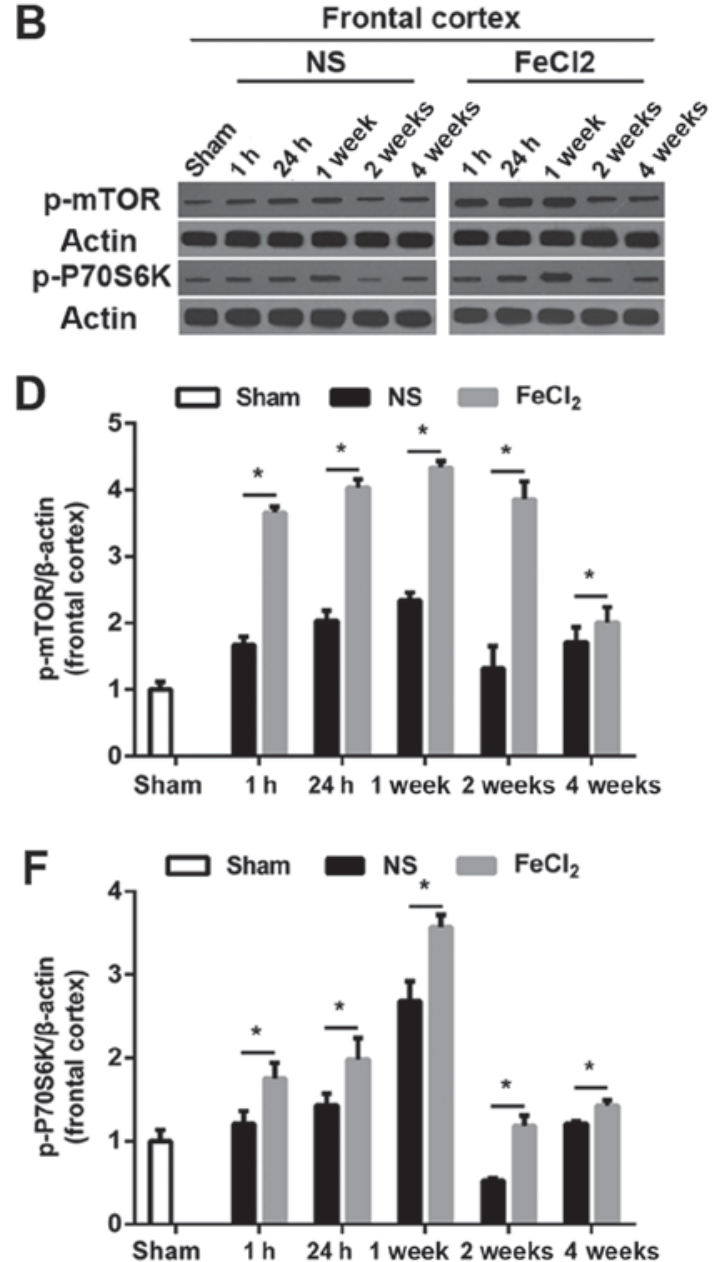

Figure 3. Temporal course of p-mTOR and p-P70S6K expression in hippocampus and frontal cortex. (A and B) Representative western blot bands of p-mTOR and p-P70S6K expression. (C-F) Quantitative results of p-mTOR and p-P70S6K expression in hippocampus and frontal cortex. Values were shown as the fold changes compared with the sham group. $\mathrm{N}=6$ per group. "P<0.05 vs. the NS-injected group. NS, normal saline; mTOR, mammalian target of rapamycin.

with rapamycin (Fig. 5; P<0.05). Additionally, there still existed slight disruptions of cellular arrangement and structure in the PTE + vehicle group, while which were also alleviated greatly both in frontal lobe and hippocampus after rapamycin treatment (Fig. 6A-D).

Rapamycin decreased the level of p-mTOR and p-P70S6K in rats after PTE induction. As predicted, the levels of $\mathrm{p}-\mathrm{mTOR}$ and p-P70S6K were obviously increased in frontal lobe and hippocampus in the vehicle group. Both proteins were significantly reduced in the PTE + rapamycin group as compared to the PTE + vehicle group (except hippocampal p-P70S6K level at 2 weeks). Specifically, perilesional and hippocampal p-mTOR levels decreased significantly at all time points after rapamycin treatment. Similar changes were found in the levels of perilesional p-P70S6K. Moreover, hippocampal p-P70S6K levels at 1 and $24 \mathrm{~h}$, as well as 1 and 4 weeks were also decreased significantly after rapamycin treatment in addition to that at 2 weeks (Figs. 7 and 8).

\section{Discussion}

The main finding of this study was that the levels of p-mTOR and p-P70S6K, biomarkers of activated mTOR signaling, increased significantly in rats subjected to PTE induction, which was accompanied by obvious behavioral seizures and cellular injury. Of interest, both elevated indicators were decreased remarkably after rapamycin treatment. Moreover, inhibition of mTOR with rapamycin also greatly reduced behavioral seizures over a longer time-period as compared to previously studies $(12,19)$. Our results suggest that the over activation of mTOR signaling may be a crucial pathogenic mechanism participated in the development of $\mathrm{FeCl}_{2}$-induced PTE. Rapamycin provides a antiepileptogenic effect through the inhibition of mTOR signaling.

At present, there are several animal models of PTE, such as controlled cortical impact model $(19,22)$, low $\mathrm{Mg}^{2+}$ model (23), cortical undercut model (24), and $\mathrm{FeCl}_{2}$-induced model (16). In the present study, we adopted the frontal cortex injection of $\mathrm{FeCl}_{2}$-induced PTE model for the following reasons: i) Frontal cortex damage is the most common type of injuries in clinical practice and the pathological change caused by $\mathrm{FeCl}_{2}$ injection is similar to human TBI (17); ii) The model has high success rate, stability and repeatability; and iii) The latency of seizures is short and beneficial to behavioral evaluation and pharmacological intervention. Most of the reasons were further verified in our study. For example, $85.2 \%$ rats developed to epileptogenesis after injection of $\mathrm{FeCl}_{2}$. Moreover, behavioral seizures 


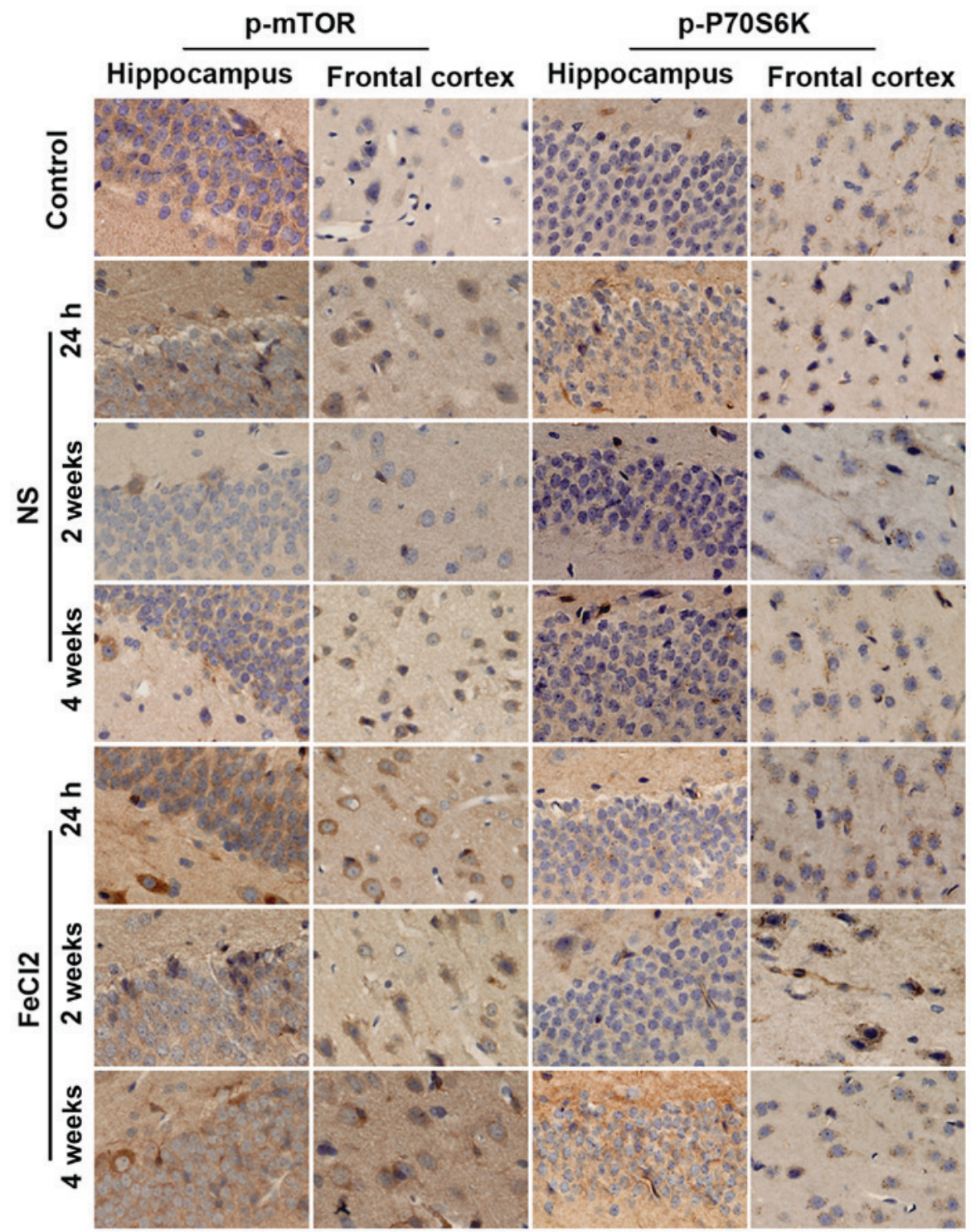

Figure 4. Time course of p-mTOR and p-P70S6K expression in hippocampus and frontal cortex. Representative immunohistochemical pictures of p-mTOR and p-P70S6K expression in the sham rats, the NS-injected rats and the $\mathrm{FeCl}_{2}$-injected rats at different time points. NS, normal saline; mTOR, mammalian target of rapamycin.

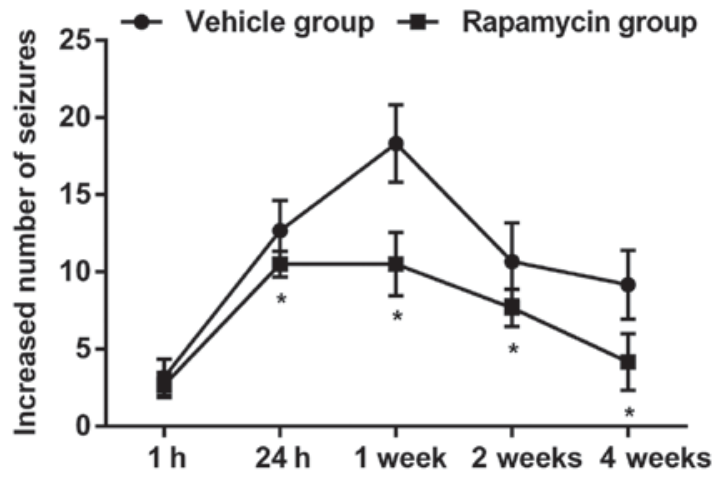

Figure 5. Antiepileptogenic effect of rapamycin. The frequency and number of behavioral seizures were significantly reduced after rapamycin treatment in the PTE + rapamycin group as compared to the vehicle group. ${ }^{*} \mathrm{P}<0.05$ vs. the vehicle group. PTE, post-traumatic epilepsy.

could be induced within as early as an hour and sustained at least 4 weeks after $\mathrm{FeCl}_{2}$ injection.
The mTOR pathway has been reported to involve in several neurological diseases including depressive disorder $(25)$, ischemia brain injury $(11,26)$, brain tumor (27) and epilepsy (28). Here, we provide evidence that the expression of p-mTOR and p-P70S6K, over activation biomarkers of mTOR signaling, strengthened heavily in hippocampus and frontal cortex after PTE induction. Consistence with our results, a study in vitro also verified the upregulation of mTOR signaling after the induction of PTE despite of without investigating the downstream proteins. In addition, literature concerning other type of epilepspy also determined the hyperactivation of mTOR and further found the increased expression of downstream proteins, such as p-P70S6K (28). PTE is a common sequela following craniocerebral injury. However, there is limited effectivity for its treatment. In recent years, rapamycin has been widely applied in experimental researches and neuroprotective effects are found in a number of diseases including TBI (29), hypoxic-ischemic encephalopathy (26), 


\section{Hippocampus}
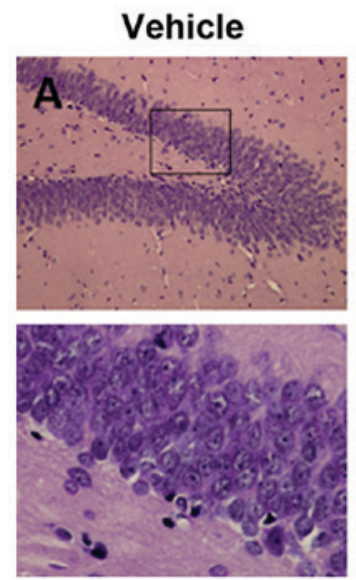

Rapamycin
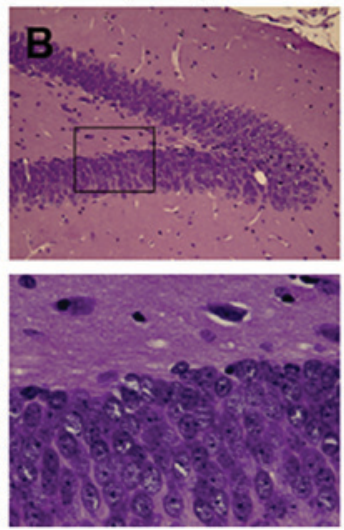

Frontal cortex

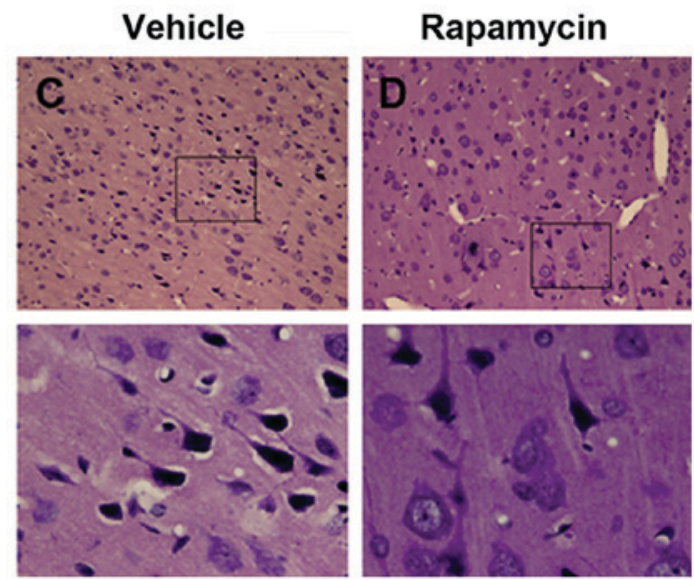

Figure 6. Effect of rapamycin on cellular injury. (A) There still existed slight impairments of cellular arrangement and structure in the vehicle group. (B) Cellular injuries in hippocampus were alleaviated greatly after rapamycin treatment. (C) There still existed slight impairments in frontal lobe after treated with vehicle. (D) Cellular injuries in frontal lobe were alleaviated greatly after rapamycin treatment. Bottom graphs (magnification, $\mathrm{x} 400$ ) are enlarged view of the boxs above, respectively.

A
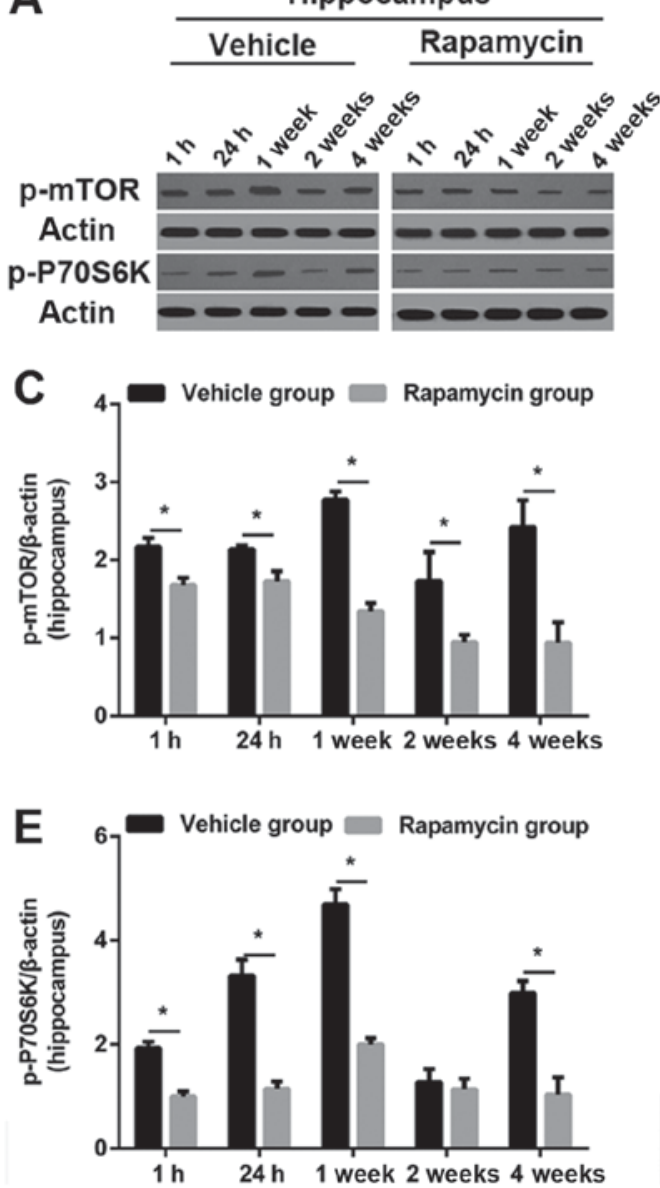

B

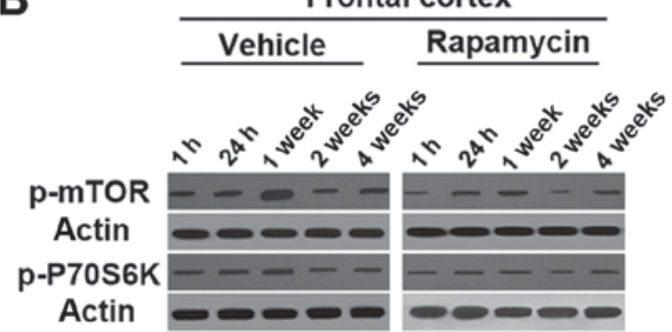

D
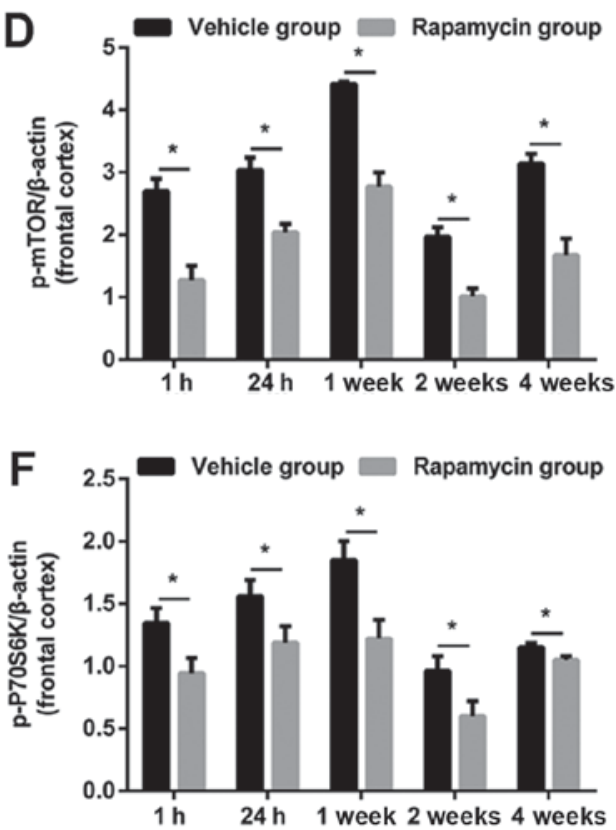

Figure 7. Effect of rapamycin on mTOR signaling assessment with western blot. (A and B) Representative western blot bands of p-mTOR and p-P70S6K expression after PTE induction with or without rapamycin treatment. (C-F) Quantitative results of p-mTOR and p-P70S6K in hippocampus and frontal cortex after vehicle or rapamycin treatment. Values were shown as the fold-changes compared to the sham group in experiment 1 . $\mathrm{N}=6$ per group. ${ }^{*} \mathrm{P}<0.05$ vs. the vehicle group. mTOR, mammalian target of rapamycin; PTE, post-traumatic epilepsy.

and epilepsy $(28,30)$. In line with the results of these studies, our results indicate that the increased levels of p-mTOR and p-P70S6K were reduced significantly after rapamycin treatment. That is, the over activation of mTOR pathway was markedly inhibited after administration of rapamycin. Meanwhile, the frequency and number of 
p-mTOR

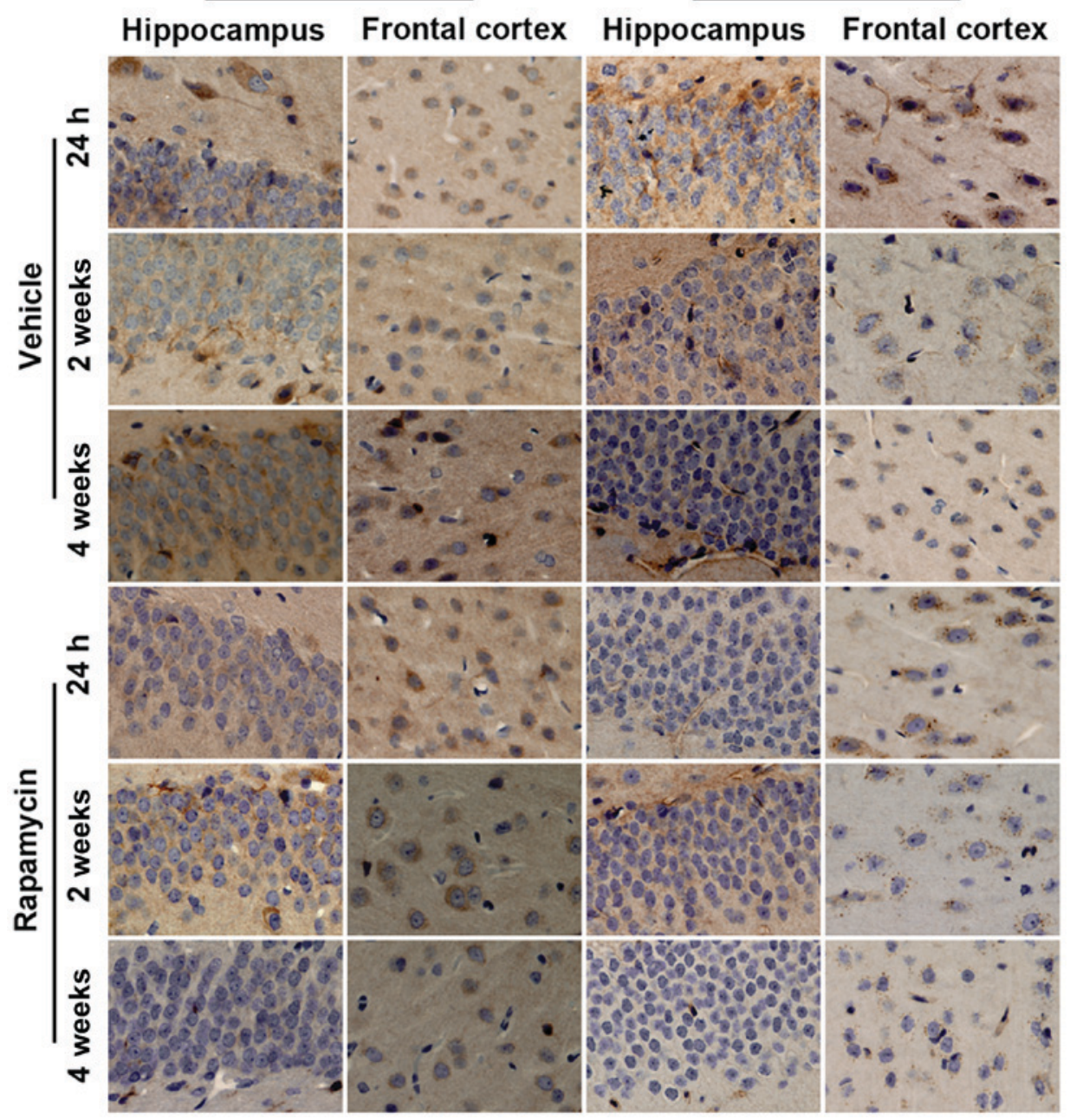

Figure 8. Effect of rapamycin on mTOR signaling assessment with immunohistochemistry. Representative immunohistochemical pictures of p-mTOR and p-P70S6K in rats after PTE induction with or without rapamycin treatment. mTOR, mammalian target of rapamycin; PTE, post-traumatic epilepsy.

behavioral seizures and epileptic injury were also greatly weakened.

Multiple potential mechanisms may contribute to the antiepileptogenic effects of rapamycin. First, hyperactivation of $\mathrm{mTOR} / \mathrm{P} 70 \mathrm{~S} 6 \mathrm{~K}$ signaling promotes oligodendrocyte proliferation and myelination (25), which is essential to the axonal sprouting susceptible to the development of epilepsy (2). Therefore, antiepileptogenic effect was obtained via blocking the mTOR signaling and decreasing the expression of downstream p-P70S6K. Second, induction of synaptic plasticity, resulting from the elevated proteins synthesis in hippocampus following activation of mTOR signaling is a important mechanism for epileptogenesis (31). Rapamycin weaken the frequency and number of seizures through decreasing the levels of certain translation initiation factors and reducing the rate of protein synthesis. Third, as previously reported (32), rapamycin reduce the activation of microglia, a hallmak of neuroinflammation, which can be attibuted to its intrinsic anti-inflammatory effect. Last but no least, although the role of mTOR signaling on neuronal apoptosis is controversial, several studies have demonstrated that inhibition of seizure-induced
mTOR signaling with rapamycin could reduce the amount of apoptosis (33).

Meanwhile, we need to notice the limitations of our study. First, electrophysiological examination is still the golden standard for epilepsy diagnosis and is crucial for the response evaluation. So it should be included in the future study. Second, the distribution of p-mTOR and p-P70S6K have not been illustrated in this study. Further tests would be conducted to identify the specific cell types that express the abovementioned biomarkers of activated mTOR signaling.

In summary, our results suggest that there exists over activation of mTOR signaling in a rat model of $\mathrm{FeCl} 2$-induced PTE. Inhibition of mTOR signaling with rapamycin significantly reduces the behavioral seizures and then ameliorates epileptic brain injury. It suggests that mTOR signaling pathway may be a potential therapeutic target, and rapamycin can be considered as a promising agent for PTE treatment.

\section{Acknowledgements}

This study was sponsored by the Natural Science Foundation of Fujian Province (no. 2015J01387), and the Medical and 
Health Science and Technology Innovation Program of Fujian Province (no. 2014-CXB-14), and the Key Clinical Specialty Discipline Construction program of Fujian, China (no. 2012-SLCZD-2).

\section{References}

1. Gupta PK, Sayed N, Ding K, Agostini MA, Van Ness PC, Yablon S, Madden C, Mickey B, D'Ambrosio R and Diaz-Arrastia R: Subtypes of post-traumatic epilepsy: Clinical, electrophysiological, and imaging features. J Neurotrauma 31: $1439-1443,2014$

2. Bhuyan P, Patel DC, Wilcox KS and Patel M: Oxidative stress in murine Theiler's virus-induced temporal lobe epilepsy. Exp Neurol 271: 329-334, 2015.

3. Irimia A and Van Horn JD: Epileptogenic focus localization in treatment-resistant post-traumatic epilepsy. J Clin Neurosci 22 627-631, 2015.

4. Christensen J, Pedersen MG, Pedersen CB, Sidenius P, Olsen J and Vestergaard M: Long-term risk of epilepsy after traumatic brain injury in children and young adults: A population-based cohort study. Lancet 373: 1105-1110, 2009.

5. Laplante M and Sabatini DM: mTOR signaling in growth control and disease. Cell 149: 274-293, 2012.

6. Wong M: Mammalian target of rapamycin (mTOR) pathways in neurological diseases. Biomed J 36: 40-50, 2013.

7. Cho $\mathrm{CH}$ : Frontier of epilepsy research-mTOR signaling pathway. Exp Mol Med 43: 231-274, 2011.

8. Crino PB: The mTOR signalling cascade: Paving new roads to cure neurological disease. Nat Rev Neurol 12: 379-392, 2016.

9. Nguyen LH, Brewster AL, Clark ME, Regnier-Golanov A, Sunnen CN, Patil VV, D'Arcangelo G and Anderson AE: mTOR inhibition suppresses established epilepsy in a mouse model of cortical dysplasia. Epilepsia 56: 636-646, 2015.

10. Sha LZ, Xing XL, Zhang D, Yao Y, Dou WC, Jin LR, Wu LW and $\mathrm{Xu} \mathrm{Q}$ : Mapping the spatio-temporal pattern of the mammalian target of rapamycin (mTOR) activation in temporal lobe epilepsy. PLoS One 7: e39152, 2012.

11. Liu P, Yang X, Hei C, Meli Y, Niu J, Sun T and Li PA: Rapamycin reduced ischemic brain damage in diabetic animals is associated with suppressions of mTOR and ERK1/2 Signaling. Int J Biol Sci 12: 1032-1040, 2016.

12. Berdichevsky Y, Dryer AM, Saponjian Y, Mahoney MM, Pimentel CA, Lucini CA, Usenovic M and Staley KJ: PI3K-Akt signaling activates mTOR-mediated epileptogenesis in organotypic hippocampal culture model of post-traumatic epilepsy. J Neurosci 33: 9056-9067, 2013

13. Sancho-Rieger J and Parra-Martinez J: Preventive and therapeutic attitude in post-traumatic epileptic seizures. Rev Neurol 35 (Suppl 1): S39-S42, 2002 (In Spanish).

14. Talos DM, Sun H, Zhou X, Fitzgerald EC, Jackson MC, Klein PM, Lan VJ, Joseph A and Jensen FE: The interaction between early life epilepsy and autistic-like behavioral consequences: A role for the mammalian target of rapamycin (mTOR) pathway. PLoS One 7: e35885, 2012.

15. Galanopoulou AS, Gorter JA and Cepeda C: Finding a better drug for epilepsy: The mTOR pathway as an antiepileptogenic target. Epilepsia 53: 1119-1130, 2012.

16. Willmore LJ and Triggs WJ: Effect of phenytoin and corticosteroids on seizures and lipid peroxidation in experimental posttraumatic epilepsy. J Neurosurg 60: 467-472, 1984.

17. Ueda Y, Willmore J and Triggs WJ: Amygdalar injection of $\mathrm{FeCl} 3$ causes spontaneous recurrent seizures. Exp Neurol 153: 123-127, 1998.
18. Racine RJ: Modification of seizure activity by electrical stimulation. II. Motor seizure. Electroencephalogr Clin Neurophysiol 32: 281-294, 1972.

19. Butler CR, Boychuk JA and Smith BN: Effects of rapamycin treatment on neurogenesis and synaptic reorganization in the dentate gyrus after controlled cortical impact injury in mice. Front Syst Neurosci 9: 163, 2015.

20. Rankin-Gee EK, McRae PA, Baranov E, Rogers S, Wandrey L and Porter BE: Perineuronal net degradation in epilepsy. Epilepsia 56: 1124-1133, 2015.

21. Zhou C, Xie G, Wang C, Zhang Z, Chen Q, Zhang L, Wu L, Wei Y, Ding H, Hang C, et al: Decreased progranulin levels in patients and rats with subarachnoid hemorrhage: A potential role in inhibiting inflammation by suppressing neutrophil recruitment. J Neuroinflammation 12: 200, 2015.

22. Kelly KM, Miller ER, Lepsveridze E, Kharlamov EA and Mchedlishvili Z: Posttraumatic seizures and epilepsy in adult rats after controlled cortical impact. Epilepsy Res 117: 104-116, 2015.

23. Gong XW, Li JB, Lu QC, Liang PJ and Zhang PM: Effective connectivity of hippocampal neural network and its alteration in Mg2+-free epilepsy model. PLoS One 9: e92961, 2014.

24. Chauvette S, Soltani S, Seigneur J and Timofeev I: In vivo models of cortical acquired epilepsy. J Neurosci Methods 260: 185-201, 2016.

25. Lloyd BA, Hake HS, Ishiwata T, Farmer CE, Loetz EC, Fleshner M, Bland ST and Greenwood BN: Exercise increases mTOR signaling in brain regions involved in cognition and emotional behavior. Behav Brain Res 323: 56-67, 2017.

26. Lee HJ, Koh SH, Song KM, Seol IJ and Park HK: The $\mathrm{Akt} / \mathrm{mTOR} / \mathrm{p} 70 \mathrm{~S} 6 \mathrm{~K}$ pathway is involved in the neuroprotective effect of erythropoietin on hypoxic/ischemic brain injury in a neonatal rat model. Neonatology 110: 93-100, 2016.

27. Fan W, Wang W, Mao X, Chu S, Feng J, Xiao D, Zhou J and Fan S: Elevated levels of p-Mnk1, p-eIF4E and p-p70S6K proteins are associated with tumor recurrence and poor prognosis in astrocytomas. J Neurooncol 131: 485-493, 2017.

28. Lippman-Bell JJ, Rakhade SN, Klein PM, Obeid M, Jackson MC, Joseph A and Jensen FE: AMPA receptor antagonist NBQX attenuates later-life epileptic seizures and autistic-like social deficits following neonatal seizures. Epilepsia 54: 1922-1932, 2013.

29. Ding K, Wang H, Wu Y, Zhang L, Xu J, Li T, Ding Y, Zhu L and $\mathrm{He} \mathrm{J}$ : Rapamycin protects against apoptotic neuronal death and improves neurologic function after traumatic brain injury in mice via modulation of the mTOR-p53-Bax axis. J Surg Res 194: 239-247, 2015.

30. Buckmaster PS and Wen X: Rapamycin suppresses axon sprouting by somatostatin interneurons in a mouse model of temporal lobe epilepsy. Epilepsia 52: 2057-2064, 2011.

31. Stoica L, Zhu PJ, Huang W, Zhou H, Kozma SC and Costa-Mattioli M: Selective pharmacogenetic inhibition of mammalian target of Rapamycin complex I (mTORC1) blocks long-term synaptic plasticity and memory storage. Proc Natl Acad Sci USA 108: 3791-3796, 2011.

32. van Vliet EA, Otte WM, Wadman WJ, Aronica E, Kooij G, de Vries HE, Dijkhuizen RM and Gorter JA: Blood-brain barrier leakage after status epilepticus in rapamycin-treated rats II: Potential mechanisms. Epilepsia 57: 70-78, 2016.

33. Zeng LH, Rensing NR and Wong M: The mammalian target of rapamycin signaling pathway mediates epileptogenesis in a model of temporal lobe epilepsy. J Neurosci 29: 6964-6972, 2009.

This work is licensed under a Creative Commons Attribution-NonCommercial-NoDerivatives 4.0 International (CC BY-NC-ND 4.0) License. 